\title{
Erratum to: Two-dimensional linear-combination model fitting of magnetic resonance spectra to define the macromolecule baseline using FiTAID, a fitting tool for arrays of interrelated datasets
}

\author{
Daniel G. Q. Chong • Roland Kreis • \\ Christine S. Bolliger • Chris Boesch • \\ Johannes Slotboom
}

Published online: 3 May 2011

(c) ESMRMB 2011

Erratum to: Magn Reson Mater Phy

DOI:10.1007/s10334-011-0246-y

Due to an error in printing style of a footnote index, the indication of a limiting condition after Eq. 16 was misleading.

The sentence should read:

Note that the indices should fulfill the conditions $D \geq 2$ and $E+F+G \geq 2^{2}$ in order to justify using these patterns, otherwise, they can be replaced by simpler forms.

With ${ }^{2}$ refering to footnote 2 .

\footnotetext{
${ }^{2}$ Not all entity types have to be present. For missing entities, the corresponding maximum index is symbolically set to 0 and the term eliminated from the summation.
}

The online version of the original article can be found under doi:10.1007/s10334-011-0246-y.

D. G. Q. Chong · R. Kreis $(\varangle) \cdot$ C. S. Bolliger · C. Boesch Department of Clinical Research, Unit for MR-Spectroscopy and Methodology, University Bern, Inselspital, P.O. Box 35, 3010 Bern, Switzerland

e-mail: roland.kreis@insel.ch

J. Slotboom

Department of Radiology, Neuroradiology, and Nuclear Medicine,

University Hospital Bern, 3010 Bern, Switzerland 